ДИАБЕТИЧЕСКАЯ НЕФРОПАТИЯ У БЕРЕМЕННЫХ С САХАРНЫМ ДИАБЕТОМ 1 ТИПА. СОВРЕМЕННЫЙ ВЗГЛЯД НА ПРОБЛЕМУ

(С.С. Шилова ${ }^{1,2 *}$, Н.В. Боровик ${ }^{1}$, М.И. Ярмолинская ${ }^{1,3}$

${ }^{1}$ Научно-исследовательский институт акушерства, гинекологии и репродуктологии им. Д.О. Отта, Санкт-Петербург

${ }^{2}$ Национальный медицинский исследовательский центр им. В.А. Алмазова, Санкт-Петербург

${ }^{3}$ Северо-Западный государственный медицинский университет им. И.И. Мечникова, Санкт-Петербург

Диабетическая нефропатия (ДН) - специфическое поражение почек при сахарном диабете (СД), которое относительно часто встречается у беременных с прегестационным СД $(5,9-26 \%)$ и является одним из основных факторов, препятствующих благоприятному течению беременности у этой группы женщин. Выраженная ДН увеличивает риски неблагоприятных исходов беременности как для матери, так и для плода, способствуя прогрессированию хронической болезни почек (ХБП), развитию преэклампсии, большей частоте преждевременных родов, оперативного родоразрешения, повышению перинатальной смертности и неонатальной заболеваемости. В то же время в мире продолжает накапливаться опыт относительно благополучных исходов беременности у женщин с тяжелой ХБП.

В данном обзоре систематизируется мировой и российский опыт планирования и ведения беременности у женщин с СД 1 типа и ДН разной степени выраженности. Обсуждаются значение нефропротективной терапии на прегравидарном этапе, достижения и поддержания целевых значений артериального давления и гликемии, необходимость наблюдения междисциплинарной командой специалистов для улучшения течения беременности и исхода родов у данной категории больных.

КЛЮЧЕВЫЕ СЛОВА: сахарный диабет 1 типа; диабетическая нефропатия; прегравидарная подготовка; беременность

\title{
DIABETIC NEPHROPATHY IN TYPE 1 DIABETES AND PREGNANCY. MODERN VIEW OF THE PROBLEM
}

\author{
(c) Ekaterina S. Shilova ${ }^{1,2 *}$, Natalya V. Borovik ${ }^{1}$, Maria I. Yarmolinskaya ${ }^{1,3}$
}

'The Research Institute of Obstetrics, Gynecology, and Reproductology named after D.O. Ott, Saint Petersburg, Russia

${ }^{2}$ Almazov National Medical Research Center, Saint Petersburg, Russia

${ }^{3}$ North-Western State Medical University named after I.I. Mechnikov, Saint Petersburg, Russia

Diabetic nephropathy (DN) is specific kidney damage in patients with diabetes mellitus. DN develops relatively often in pregestational diabetes patients (5,9-26\%) and stills one of the main limitations for successful pregnancy in this patients' group. Advanced DN increases risks of poor pregnancy outcomes for women and fetuses including chronic kidney disease (CKD) progression, high rate of preeclampsia, preterm deliveries, Cesarean sections, perinatal mortality and neonatal morbidity. At the same time there are more and more successful pregnancies with advanced DN in the wold.

In our paper we systematize global experience of planning and management pregnancies with type 1 diabetes and DN in different stages of renal impairment. We discuss role of nephroprotective therapy in preconception care, achievement and maintaining blood pressure goals, multidisciplinary team care for improvement pregnancy outcomes in type 1 diabetic women with DN.

KEYWORDS: type 1 diabetes mellitus; diabetic nephropathy; preconception care; pregnancy

Диабетическая нефропатия (ДН) - специфическое поражение почек при сахарном диабете (СД), сопровождающееся формированием узелкового гломерулосклероза с последующим возможным развитием терминальной почечной недостаточности [1]. ДН представляет собой частный случай хронической болезни почек (ХБП), соответственно, диагностируется при наличии доказанных нарушений структуры или функции почек, существующих не менее 3 месяцев, и стадируется в соответствии с уровнем скорости клубочковой фильтрации (СКФ) С1-С5 и альбуминурии А1-А3 [2].

ДН встречается у 5,9-26\% беременных с прегестационным СД [3-7]. В Дании распространенность ДН в стадии протеинурии среди беременных с СД 1 типа (СД1) составила 2,5\%, микроальбуминурия была выявлена у 3,4\% [3]. В крупном британском исследовании (более 750 участников) микроальбуминурия встречалась у 4,5\%, протеинурия - у 1,9\% беременных с СД1 [4]. 
Ekbom и соавт. зафиксировали ДН у 16\% беременных с СД1, из них микроальбуминурия была выявлена в $11 \%$ случаев, протеинурия - в 5\% случаев [5]. В России распространенность ДН среди женщин репродуктивного возраста составила $26,1 \%$, из них у $14,9 \%$ имела место микроальбуминурия и у 11,2\% - протеинурия [6]. В исследовании Klemetti и соавт. частота выявления протеинурии среди беременных женщин снизилась с 14,7\% в 1988-1999 гг. до 6,5\% в 2000-2011 гг. [7]. Вероятно, снижение частоты ДН среди беременных женщин объясняется более жестким контролем гликемии и артериальной гипертензии (АГ) до наступления беременности, а также своевременным назначением препаратов, блокирующих ренин-ангиотензин-альдостероновую систему (РАAC).

Во время беременности происходят значительные гемодинамические изменения, приводящие к увеличению частоты сердечных сокращений, минутного объема и сердечного выброса, увеличению объема циркулирующей крови и СКФ на 40-60\%, возрастанию диастолического артериального давления (АД). Наиболее частым осложнением беременности у больных СД является преэклампсия, в патогенезе которой важная роль принадлежит нарушениям микроциркуляции. Все это может способствовать прогрессированию микрососудистых диабетических осложнений (ДН и диабетической ретинопатии (ДР)). Кроме того, дислипидемия и гипертензивные нарушения, часто присоединяющиеся во второй половине беременности, ухудшают функцию почек. ДН - один из основных факторов, препятствующих наступлению и успешному течению беременности при СД за счет рисков для здоровья как матери, так и плода.

\section{РИСКИ ДЛЯ МАТЕРИ}

Данные о влиянии беременности на течение ДН ограничены. Беременность, вероятно, не повышает риск развития ДН у женщин без патологии почек [8]. Однако в ретроспективном исследовании Biesenbach и соавт. было выявлено, что у женщин с СД1 без патологии почек, но с развившейся преэклампсией чаще формировалась ДН в течение 11 лет наблюдения, чем у женщин с неосложненным течением беременности [9].

Большинство исследователей отмечают временное ухудшение почечной функции во время беременности у женщин с начальными проявлениями ДН, однако после родоразрешения уровень креатинина в крови и протеинурии возвращался к изначальным значениям $[6,10,11]$. В исследовании DССТ более 90\% беременных женщин имели суточную потерю альбумина в пределах нормальных значений, при этом максимальный уровень альбуминурии в группе интенсивной терапии инсулином был достигнут к III триместру беременности (OP 1,59; $p=0,02)$, а через год после родов уже практически не отличался от исходного (ОР 1,18; $\mathrm{p}=0,02)$ [11]. Боровик Н.В. и соавт. отмечают увеличение суточной протеинурии у 20\% женщин с СД1 в III триместре беременности. Усиление протеинурии наблюдалось преимущественно у больных с исходной ДН (у 50\% больных с микроальбуминурией и у 53,3\% больных с протеинурией до беременности). При обследовании функции почек через 6 месяцев после родов различий в динамике микроальбуминурии, суточной протеинурии, уровне креатинина в крови и СКФ выявлено не было (p>0,05) [6]. В отдаленной перспективе при оценке функции почек также не было различий у женщин с микроальбуминурией, имевших и не имевших беременности в анамнезе [8, 12].

У женщин с выраженной ДН прогноз менее благоприятный. Протеинурия прогрессирует в течение беременности и к концу III триместра более $70 \%$ женщин с ДН в стадии протеинурии достигают потери белка более 3 г/сут $[13,14]$. В исследовании Gordon и соавт. уженщин с СД1 и ДН (СКФ менее 90 мл/мин/1,73 м² и/или протеинурия более 1 г/сут) в I триместре беременности отмечалось прогрессирование ДН после родов [15]. В работе Klemetti и соавт. суточная протеинурия у женщин с ДН до беременности составляла 0,80 $(0,34-4,0)$ г, прогрессивно нарастала со сроком беременности и достигала к III триместру высоких значений - 4,22 (0,45-19,80) г/сут [7]. Предполагается, что ДН имеет более неблагоприятное течение на фоне беременности в сравнении с другими формами нефропатии, при которых ухудшение почечной функции после родов ассоциировано со СКФ <40 мл/мин/1,73 м² и протеинурией более 1 г/сут [16].

Данные о влиянии беременности на развитие терминальной почечной недостаточности в литературе представлены мало. Большинство исследований проводилось в 1980-1990 х гг. на небольшой выборке пациентов и в условиях других подходов к терапии диабета и технических возможностей контроля гликемии. В работах Biesenbach и соавт. и Purdy и соавт. были получены неутешительные результаты о быстром прогрессировании ХБП у женщин с выраженной ДН во время беременности $[17,18]$. При наблюдении за 5 пациентками с ДН (за 3 месяца до зачатия СКФ 37-75 мл/мин/1,73 м², протеинурия 2,1-4,0 г/сут) у всех женщин потребовалось проведение диализа в течение 42 месяцев после родов [17]. Согласно результатам другого исследования, из 11 пациенток с уровнем креатинина >124 мкмоль/л в начале беременности 7 потребовалось начать терапию диализом в течение 27士16,4 месяцев наблюдения после родов, при этом у двух из них функция почек во время беременности оставалась стабильной [18]. Другие исследователи получили более благоприятные результаты. Mackie и соавт. разделили 22 беременных с СД1 и ДН на две группы. В первую группу вошли 10 пациенток с уровнем креатинина в крови >125 мкмоль/л, во вторую - 12 пациенток с протеинурией, но сохранной функцией почек. Должная компенсация углеводного обмена в обеих группах отсутствовала (уровень гликированного гемоглобина $\left(\mathrm{HbA}_{1 c}\right) 12,6 \%$ (8-16\%) и 10,6\% (7,4-16,4\%) соответственно). Беременность была прервана до 20-й недели у 4 женщин из 1-й группы (3 самопроизвольных выкидыша и 1 искусственное прерывание беременности в связи с множественными пороками развития) и у 1 женщины из 2-й группы (искусственное прерывание беременности в связи с множественными пороками развития). Среди оставшихся 6 женщин из 1-й группы 3 потребовалась терапия гемодиализом в течение 0,5-8 лет наблюдения после родов, тогда как во 2-й группе гемодиализ был применен только к одной 
пациентке [19]. По данным Gordon и соавт., у 7 женщин с уровнем СКФ 60-90 мл/мин/1,73 м² (76 18$)$ и у 4 жен-

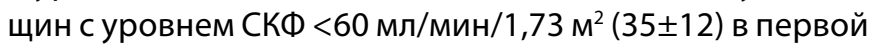
половине беременности после ее завершения уровень

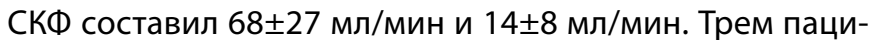
енткам с изначальным уровнем СКФ $<60$ мл/мин/1,73 м² потребовались терапия диализом и пересадка почки через 8, 15 и 41 месяц после родов. Компенсация гликемии на прегравидарном этапе и во время беременности в данном исследовании не оценивалась [15]. В Дании не было получено различий в отдаленных исходах (наблюдение в течение 3-28 лет) у женщин с СД1 и ДН, имевших и не имевших в анамнезе беременность. Все пациентки получали антигипертензивную терапию, целевыми значениями считались цифры АД <140/90 мм рт.ст. В течение наблюдения 35\% женщин, имевших беременность, умерли, а 19\% достигли терминальной стадии почечной недостаточности, по сравнению с 34\% и 24\% соответственно во 2-й группе (p>0,05) [20]. В более позднем исследовании Piccoli и соавт. из 12 беременных с СД1 и нефропатией СКФ <60 мл/мин/1,73 м² в I триместре беременности имела место у 3, а в III триместре у 5 женщин. Терминальная почечная недостаточность развилась у 2 пациенток через 1 и через 3 года соответственно [21].

Женщины с СД1 и тяжелой ДН имеют значительный риск заболеваемости и смертности в отдаленной перспективе, что также необходимо иметь в виду и обсуждать в контексте возможного материнства. По данным двух различных исследований, 35\% (9/25) [7] и 16\% (12/108) [20] женщин с СД1 и ДН в стадии протеинурии умерли в течение 16 и 25 лет наблюдения соответственно.

\section{РИСКИ БЕРЕМЕННОСТИ}

Установлено, что у женщин с прегестационным диабетом неонатальные исходы хуже, чем в общей популяции [22]. Однако значимые различия в неонатальных исходах беременностей у женщин с СД при отсутствии или наличии нефропатии получены не во всех исследованиях [3, 23].

Многочисленные исследования подтвердили прямую связь между декомпенсированным СД и частотой развития врожденных пороков развития (ВПР) плода. Чем выше уровень $\mathrm{HbA}_{1 c^{\prime}}$ тем выше частота ВПР плода, достигая 25\% при уровне $\mathrm{HbA}_{1 с}$ более $10 \%$. По данным Bell и соавт., ДН повышает риск формирования значимых нехромосомных ВПР плода вне зависимости от уровня $\mathrm{HbA}_{1 c}$ (ОШ 2,5 [95\% ДИ 1,1-5,3]). При этом риск ВПР ожидаемо линейно возрастал при повышении $\mathrm{HbA}_{1 с}$ выше 6,3\% (на 30\% при повышении $\mathrm{HbA}_{1 с}$ на 1\%) [23]. С чем связан повышенный риск ВПР при ДН, не вполне ясно. ДН, как правило, является следствием длительно неконтролируемого СД, со значимой вариабельностью гликемии, тогда как $\mathrm{HbA}_{1 c}$ не всегда является истинным отражением компенсации СД [24]. Однако другие осложнения СД, такие как ДР и нейропатия, ассоциации с ВПР не показали [23]. Возможной причиной может быть и терапия ингибиторами ангиотензинпревращающего фермента (иАПФ), не отмененная своевременно при незапланированной беременности [25]. По некоторым данным, существует генетическая предрасположенность к развитию ДН, возможно, она способствует более высокому риску ВПР [26]. Оксидативный стресс может играть роль как в формировании ДН, так и ВПР [27]. Как следствие, существует мнение, что женщинам с ДН может быть рекомендован прием больших доз фолиевой кислоты с этапа планирования и в I триместре беременности (5 мг/сут) [28].

К факторам риска развития преэклампсии относятся предшествующая беременности АГ, наличие ДН, неудовлетворительный гликемический контроль до 20-й недели беременности. Преэклампсия, по разным источникам, встречается у 35-64\% беременных с ДН [5, 6, 29], в то время как у женщин с СД без ДН преэклампсия имеет место только в 9-17\% случаев [30, 31, 32]. По данным Jensen и соавт., микроальбуминурия в I триместре является значимым предиктором развития преэклампсии (ОШ 4,0; [95\% ДИ 2,2-7,2]), так же как и уровень $\mathrm{HbA}_{1 c}$ в III триместре беременности (ОШ 1,3; [95\% ДИ 1,1-1,5] на 1\% повышения $\mathrm{HbA}_{1 c}$ ) [29]. Вероятно, связь преэклампсии и микроальбуминурии обусловлена эндотелиальной дисфункцией. Например, у женщин с СД и другими признаками эндотелиальной дисфункции (ДР, снижение нитроглицерин-индуцированной дилатации сосудов) чаще развивается преэклампсия по сравнению с женщинами без признаков сосудистых осложнений [31]. По данным Мозговой Е.В., у беременных с преэклампсией на фоне СД1 наблюдался высокий уровень маркеров эндотелиальной дисфункции, в 2 раза превышающий данные показатели при преэклампсии без экстрагенитальной патологии $(p<0,05)$ [33]. По данным Kelly и соавт., преэклампсия встречалась статистически значимо чаще у беременных с СД1 и наличием маркеров патологии почек (повышение NGAL в моче, скорректированное по креатинину; повышение уровня СКФ, рассчитанной по формуле (KD EPI), но без клинических проявлений нефропатии (альбумин/креатинин <30 мг/г, отсутствие АГ), по сравнению с теми, кто не имел ранних маркеров повреждения почек [34]. Частота развития преэклампсии зависит от выраженности ДН. Так, у женщин, имевших микроальбуминурию до наступления беременности, преэклампсия диагностировалась в 20-40\% случаев, тогда как при исходной протеинурии этот процент составил 45-64\% [1, 29]. По данным регистра Норвегии, включившего 2204 женщины с СД, у пациенток с преэклампсией во время беременности в 4-5 раз чаще развивалась терминальная почечная недостаточность или смерть в отдаленной перспективе (20+9 лет) [35].

Вероятно, назначение аспирина целесообразно с целью профилактики преэклампсии в группе высокого риска, к которой, без сомнения, относятся женщины с ДН [28]. Американская диабетическая ассоциация (ADA) рекомендует назначение аспирина всем женщинам с СД1 и СД2 типа после 12-й недели беременности с целью профилактики преэклампсии [36]. В работах НИИ АГиР им. Отта также было показано уменьшение клинических и лабораторных проявлений преэклампсии под влиянием терапии низкомолекулярными гепаринами [33, 37].

Преждевременные роды, приводящие к повышенной неонатальной заболеваемости, также чаще встречаются у женщин с ДН. Частота преждевременных родов при ДН у больных СД1 колеблется от $11 \%$ до 70\% и зависит от степени выраженности ДН до наступления 
беременности $[29,38]$. В исследовании Klemetti и соавт. недостаточный контроль АД в I триместре среди 108 беременных с СД1 оказался независимым предиктором преждевременных родов [7]. Частота оперативного родоразрешения путем кесарева сечения у женщин с ДН составляет $60-83 \%[6,7]$.

Крупные размеры плода и ассоциированные с ними осложнения (травматизм в родах) часто встречаются у женщин с прегестационным СД без сосудистых осложнений или с начальными проявлениями микрососудистых осложнений [22], но редко регистрируются у женщин с ДН, в том числе с микроальбуминурией $[3,5]$. При наличии выраженной ДН и АГ характерна гипотрофия плода и новорожденного. По данным литературы, у 23-50\% детей отмечается синдром задержки роста плода (СЗРП) [7]. Гипотрофия встречается чаще при большей тяжести патологии почек и отражает ухудшение плацентарного кровотока [39]. Дисперсионный анализ показал зависимость массы тела новорожденного от выраженности ДР ( $\mathrm{F}=8,57$; $\mathrm{p}<0,001)$, ДН и гестоза $(F=25,32 ; p<0,001)[6]$.

\section{ПЛАНИРОВАНИЕ БЕРЕМЕННОСТИ И ПОДХОДЫ К ВЕДЕНИЮ БЕРЕМЕННОСТИ ПРИ ДИАБЕТИЧЕСКОЙ НЕФРОПАТИИ}

Преимущества планирования беременности при прегестационном СД, в том числе снижение риска ВПР, перинатальной смертности и неонатальной заболеваемости, а также экономическая оправданность прегравидарной подготовки убедительно доказаны в литературе [39, 40]. В метаанализе, включающем 12 исследований и 3088 женщин с СД, было показано, что прегравидарная подготовка снижает риск ВПР плода (ОР 0,25; [95\% ДИ 0,16-0,37]), а также риск перинатальной смертности (ОР 0,34; [95\% ДИ 0,15-0,75]) [40].

Планирование беременности у женщин с ДН является обязательным условием, позволяющим оценить выраженность почечной патологии, риски данной беременности для матери и плода и возможность их снижения за счет стабилизации АД, внутриклубочкового давления и уровня гликемии.

\section{Диагностика диабетической нефропатии}

и беременность

Диагностирование и стадирование ДН во время беременности представляет собой значимую клиническую проблему. Во-первых, для постановки диагноза необходимо два подтверждающих ДН анализа из трех, выполненных в период от 3 до 6 месяцев, что не всегда выполнимо в случае первичного обращения уже во время беременности. Во-вторых, использование расчетных формул СКФ во время беременности не рекомендовано и требует оценки клиренса эндогенного креатинина в пробе Реберга - метода, включающего сбор суточной мочи, что представляет собой неудобство для пациента, погрешности при сборе или подсчете объема мочи. В-третьих, при физиологической беременности СКФ может увеличиваться до 50\%. Соответственно, оценить истинную стадию ХБП у беременной женщины, не обследованной на прегравидарном этапе, затруднительно. Наконец, учитывая, что преэклампсия - это повышение АД в сочетании с протеинурией, развившееся после 20-й недели беременности, провести дифференциальную диагностику между ДН и преэклампсией при первом обращении женщины за медицинской помощью во второй половине беременности не представляется возможным [41]. Есть данные, что определение сывороточного уровня цистатина С и расчет с его помощью СКФ повышает надежность диагностики поражения почек у больных СД1 при нормальном уровне креатинина в сыворотке крови и отсутствии снижения СКФ, рассчитанной с использованием креатинина [42].

В любом случае пациенткам, планирующим беременность, а также на первом визите во время беременности должно быть выполнено функциональное обследование почек, включающее:

- определение отношения альбумин/креатинин в разовой, предпочтительно утренней порции мочи или альбумина в суточной моче;

определение CKФ по формуле CKD-EPI (для планирующих беременность) или клиренсовыми методами (во время беременности);

- оценка уровня АД [1].

\section{Блокаторы ренин-ангиотензин-альдостероновой} системы (PAAC)

Блокаторы РАAС положительно влияют на клубочковую фильтрацию благодаря снижению внутриклубочкового давления за счет расширения выносящей артериолы. Однако терапия блокаторами РААС во время беременности сопряжена с тератогенными эффектами. В исследовании Cooper и соавт. были обследованы 29507 детей от матерей без СД: 209 женщин получали терапию иАПФ в I триместре, 202 женщины принимали другие антигипертензивные препараты, группа контроля составила 29096 человек. В случае терапии иАПФ в I триместре у новорожденных был больший риск ВПР в сравнении с отсутствием антигипертензивной терапии (ОР 2,71; [95\% ДИ 1,72-4,27]). Применение других антигипертензивных препаратов, по данным исследователей, риск ВПР плода не увеличивало [25]. B то же время Li и соавт. в своей работе показали, что риск пороков развития сердечно-сосудистой системы плода при использовании иАПФ в I триместре беременности не превышает такой риск в случае использования других антигипертензивных препаратов в сравнении с группой контроля (ОШ 1,54; [95\% ДИ 0,90-2,62] и ОШ 1,52; [95\% ДИ 1-04-2,21] соответственно) или же просто наличия нелеченой АГ у матери [43]. Лечение иАПФ во II и III триместрах беременности ассоциировано с большим риском маловодия и патологии почек у новорожденных [44]. По мнению АДА, иАПФ и антагонисты рецепторов ангиотензина II (APA II) не должны применяться у сексуально-активных женщин репродуктивного возраста, учитывая высокую частоту незапланированных беременностей [36]. Национальный институт здоровья и клинического совершенствования Великобритании (NICE) рекомендует продолжать терапию блокаторами РААС вплоть до зачатия, чтобы минимизировать срок отсутствия лечения [45]. В таком случае женщинам с нерегулярным менструальным циклом, получающим иАПФ, стоит периодически выполнять тест на беременность, чтобы своевременно отменить 
потенциально тератогенную терапию [28]. Согласно принятым в России рекомендациям, терапия иАПФ или APA II должна быть отменена до наступления беременности, а в случае возобновления лечения после родов необходимо прервать лактацию [1].

\section{Контроль артериального давления}

Достижение целевого уровня АД с помощью своевременного назначения антигипертензивных препаратов является залогом успешной беременности у женщин с ДН [38]. По данным Klemetti и соавт., более чем у $90 \%$ женщин с ДН в стадии протеинурии регистрируется повышенное АД к III триместру беременности [7]. Carr и соавт. было продемонстрировано, что при недостаточном контроле АД (среднее АД $\geq 100$ мм рт.ст.) в сравнении с группой женщин, достигших целевого уровня АД (среднее АД <100 мм рт.ст.) уровень протеинурии $(4,69 \pm 1,08$ и 1,65 $\pm 0,43$ г/24 ч; $p=0,007)$ и креатинина в крови $(1,23 \pm 0,17$ и 0,85 $\pm 0,06$ мг/дл; $p=0,02)$ во второй половине беременности был выше, а преждевременные роды на сроке до 32-й недели беременности чаще $(38,1 \%$ и 4,6\%; $p=0,007)$. Риск преждевременных родов оставался статистически значимо выше при учете длительности диабета и уровня $\mathrm{HbA}_{1 c}$ [46]. Peкомендуемый ADA уровень АД для беременных с хронической АГ - 120-160/80-105 мм рт.ст., что позиционируется как оптимальные значения для предотвращения СЗРП. Однако для пациентов с ДН рекомендованное АД <140/90 мм рт.ст., а в случае наличия факторов риска прогрессии ХБП, в частности, альбуминурии, возможно рассмотреть АД <130/80 мм рт.ст. в качестве целевого [36]. В частности, группа ученых из Дании пришла к выводу, что инициация антигипертензивной терапии при АД >135/85 мм рт.ст. или суточной альбуминурии $\geq 300$ мг блокаторами РАAС на прегравидарном этапе или разрешенными препаратами во время беременности позволяет снизить риск преждевременных родов и преэклампсии у женщин с ДН [3, 38, 47]. Большинство экспертов сходятся во мнении, что АД ниже 130/80 мм рт.ст. является целевым во время беременности у женщин с ДН $[1,36,47]$.

Для терапии АГ во время беременности разрешены к использованию метилдопа и нифедипин, допустимо применение бета-блокаторов, но с осторожностью, учитывая возможность маскирования симптомов гипогликемии $[1,36]$.

\section{Контроль гликемии}

Достижение и поддержание целевых значений гликемии позволяют замедлить или остановить прогрессирование ДН [48-51]. В метаанализе Fullerton и соавт., включающем 5 исследований и 1475 пациентов соответственно, было получено, что интенсивный контроль гликемии статистически значимо снижает риск развития микроальбуминурии (OP=0,56; [95\% ДИ 0,46-0,68]; $\mathrm{P}<0,00001)$, однако данных в пользу замедления прогрессирования до протеинурии получено не было $(\mathrm{OP}=0,79 ;$ [95\% ДИ 0,37-1,70]) [48]. Другой метаанализ (5 исследований, 1635 пациентов) показал уменьшение суточной альбуминурии у пациентов с $\mathrm{OP}=0,37$; [95\% ДИ 0,27-0,50]; Р<0,00001) [49]. В исследовании Shurraw и соавт. принимали участие 23296 человек с СД и расчетной СКФ менее 60 мл/мин/1,73 м². При наблюдении в течение 46 месяцев было выявлено, что среди пациентов с СКФ 30-59,9 мл/мин/1,72 м² риск развития терминальной стадии ХБП был выше на $22 \%$ и 152\% при $\mathrm{HbA}_{1 с}$ более 7\% и более 9\% соответственно по сравнению с теми, у кого $\mathrm{HbA}_{1 с}$ был менее 7\% $(\mathrm{P}<0,001)$ [50]. В исследовании DССТ 1441 человек были рандомизированы на группы интенсивного и обычного контроля гликемии ( $\mathrm{HbA}_{1 c}$ 7,3\% и 9,1\% соответственно). Через 6,5 года интенсивный подход к терапии был ассоциирован с меньшим уровнем микроальбуминурии $(10,2$ и $17,7 \%$ соответственно; $\mathrm{P}<0,01)$ и протеинурии $(1,4$ и 3,2 соответственно; $\mathrm{P}<0,05)$ [51]. В исследовании Газизовой было показано, что недостаточная компенсация углеводного обмена $\left(\mathrm{HbA}_{1 c}>6,0 \%\right)$ у беременных с СД1 сопровождается повышением экскреции с мочой ранних маркеров развития диабетической нефропатии, провоспалительных цитокинов (интерлейкина-1b и моноцитарного хемоаттрактантного протеина-1) и трансформирующего фактора роста-b1 во II и III триместрах беременности [52]. Не вызывает сомнений необходимость достижения целевых значений гликемии начиная с этапа планирования беременности и на всем ее протяжении как для стабилизации течения ДН, так и для достижения лучших исходов беременности $[39,40,50]$.

\section{Диагностика и лечение инфекций мочевыводящих}

\section{путей}

Инфекции мочевыводящих путей (ИМП) чаще встречаются у женщин с СД, чем в общей популяции [53]. При этом у пациентов с ДН ИМП наблюдаются чаще, чем у пациентов без нарушений функции почек [54]. Развиваясь во время беременности, ИМП повышают риск преэклампсии, СЗР, преждевременных родов, оперативного родоразрешения путем кесарева сечения [55]. Кроме того, ИМП могут проявляться ухудшением функции почек в виде протеинурии и затруднять диагностику ДН. Настороженность в отношении ИМП на прегравидарном этапе и во время беременности имеет большое значение для достижения благоприятных исходов беременности у женщин с ДН.

\section{Диабетическая нефропатия как противопоказание} к беременности

Доказано, что при выраженной ХБП фертильность снижается. Отчасти это может быть связано с гиперпролактинемией вследствие подавления дофаминергической активности при снижении почечного клиренса $[56,57]$. Уровень ЛГ и ФСГ также повышен при ХБП, однако адекватного нарастания ЛГ в середине цикла, как правило, не происходит [58]. Тем не менее женщины, в том числе и с тяжелой ДН, планируют и могут получить беременность.

Согласно существующим в России рекомендациям, наступление беременности при выраженной нефропатии нежелательно при уровне креатинина в крови $>120$ мкмоль/л, СКФ<60 мл/мин/1,73 м², протеинурии $\geq 3$ г/сут [1]. Более того, наличие СД с тяжелой нефропатией (клиренс креатинина менее 60 мм/мин/1,73 м², суточная протеинурия 3,0 г и более, креатинин крови более 120 мкмоль/л, АГ), так же как и СД после трансплантации 
почки, являются медицинскими показаниями для искусственного прерывания беременности [59].

Согласно рекомендациям NICE, все женщины с уровнем креатинина в крови более 120 мкмоль/л, соотношением альбумин/креатинин более 30 мг/ммоль или суточной потерей белка более 0,5 г/день должны быть осмотрены нефрологом для определения тактики дальнейшего ведения [45].

Тем не менее, стоит принять во внимание, что в мире продолжает накапливаться опыт относительно благополучных исходов беременности у женщин с тяжелой ХБП [41, 56, 59]. Исследование случай-контроль было выполнено Piccoli и соавт.: были проанализированы течение и исходы 12 беременностей у женщин с СД1 и ДН, наблюдавшихся в нефроцентре г. Турина. При ранних сроках беременности у 4 пациенток СКФ была более 90 мл/мин/1,73 м², у 5 - 60-90 мл/мин/1,73 м² и у 3 - 30-60 мл/мин/1,73 м². Уровень суточной потери белка у 3 пациенток был менее 0,3 г/сут, у 5 пациенток - 0,3-3,0 г/сут, у 4 пациенток - более 3 г/сут. Нефропротективная терапия во время беременности была направлена на снижение гиперфильтрации и включала достижение контроля гликемии, целевого АД до 120-130/80 мм рт.ст. и постельный режим. На момент родоразрешения у 4 из 12 пациенток стадия ХБП достигла С3b и менее, у 9 из 12 пациенток протеинурия достигла нефротической стадии более 3 г/сут. Все беременности были завершены преждевременно, из них 7 на сроке до 34 недель беременности, тем не менее, все дети, кроме одного из близнецов, имели массу более 10-го перцентиля. Через 3 месяца после родов только у 2 пациенток СКФ осталась выше 90 мл/мин/1,73 м², у 4 СКФ соответствовала 60-90 мл/мин/1,73 м², у 3 - СКФ оказалась в диапазоне 45-60 мл/мин/1,73 м² и у 2 - была менее 30 мл/мин/1,73 м². Из них одна пациентка достигла необратимого повышения уровня креатинина и потребовала терапии диализом через 1 год после родов. Через 3 месяцапослеродов суточнаяпротеинурияболее 3 г/сут сохранялась у 4 пациенток [21]. В исследовании IbarraHernandez и соавт. принимали участие 62 беременные с ХБП (сывороточный креатинин 1,8 (1,1-3,0) мг/дл, рассчитанная СКФ 38,1 (21,9-68,0) мл/мин/1,73 м²). Диализ выполнялся 20 женщинам (2 получали диализ на прегравидарном этапе, 18 потребовали лечения диализом во время беременности), 15 из них продолжили диализ после родов [60].

Рекомендации нефрологов, однозначно запрещающие планирование и пролонгирование беременности при патологии почек, в том числе и тяжелой степени, на данный момент отсутствуют. Напротив, нефрологи отмечают высокие шансы на завершение беременности у женщин с выраженной ХБП рождением живых детей, в том числе, благодаря возможностям терапии гемодиализом $[59,61]$. Однако высокий риск такой беременности как для матери, так и для плода очевиден: прогрессирование ДН, инвалидизация детей, финансовая и психологическая нагрузка на женщину и ее близких при терапии гемодиализом $[15,21]$. Таким образом, возможность пролонгирования беременности у женщин с тяжелой дисфункцией почек может быть рассмотрена только при условии настойчивого желания женщины, контроля основного заболевания, участия междисциплинарной врачебной команды, включающей акушера-гинеколога, нефролога, эндокринолога, психолога, офтальмолога, неонатолога, доступности гемодиализа для пациентки и, конечно, понимания женщиной возможных рисков при наступлении и вынашивании такой беременности как для нее, так и для ребенка $[41,60]$.

После трансплантации почки наступление беременности также возможно. По мнению исследователей, наиболее благоприятными условиями для беременности в таком случае будут: молодой возраст, отсутствие ожирения, снижения СКФ, протеинурии, высокий комплаенс и длительность периода после трансплантации два года и более $[41,62]$. Однако KDIGO допускает возможность планирования беременности спустя год после трансплантации почки в случае протеинурии <1 г/сут [63].

\section{ЗАКЛЮЧЕНИЕ}

Давно не вызывает сомнений возможность благоприятного исхода беременности при СД. При этом риски запланированной беременности у женщин с СД1 приближаются к популяционным. Достижение целевых значений гликемии может потребовать времени и навыков, но выполнимо при достаточном уровне мотивации. Стабилизация ДР также возможна как при планировании, так и во время беременности благодаря доступности лазерной коагуляции сетчатки. Длительная терапия блокаторами РАAC (не менее 6 месяцев до зачатия) на этапе прегравидарной подготовки, достижение целевого уровня гликемии, $\mathrm{HbA}_{1 с}$ и АД с этапа планирования и во время беременности способствуют улучшению исходов беременности у больных СД1 и ДН. Несмотря на это, тяжелая ДН остается практически единственным плохо корригируемым фактором, препятствующим благополучному течению беременности при СД. Тем важнее информировать молодых пациенток с СД о необходимости контроля гликемии и планирования беременности, в том числе и в контексте развития и прогрессирования ДН и ее влияния на репродуктивную функцию. Достижение целевых значений гликемии, регулярное обследование функции почек и контроль АД позволят не допустить развития или прогрессирования ДН, а также своевременно поставить диагноз и начать лечение, тем самым увеличивая шансы на благоприятный исход беременности.

Благоприятный исход беременности у больных СД1 и ДН возможен в случае полноценной прегравидарной подготовки и специализированного наблюдения междисциплинарной командой специалистов во время беременности.

\section{ДОПОЛНИТЕЛЬНАЯ ИНФОРМАЦИЯ}

Источники финансирования. Поисково-аналитическая работа и подготовка статьи осуществлены на личные средства авторского коллектива.

Конфликт интересов. Авторы декларируют отсутствие явных и потенциальных конфликтов интересов, связанных с публикацией настоящей статьи.

Участие авторов. Шилова Е.С. - написание, редактирование и финальное утверждение рукописи; Боровик Н.В. - написание, редактирование и финальное утверждение рукописи; Ярмолинская М.И. - редактирование и финальное утверждение рукописи. 


\section{СПИСОК ЛИТЕРАТУРЫ | REFERENCES}

1. Дедов И.И., Шестакова М.В., Майоров А.Ю., и др. Алгоритмы специализированной медицинской помощи больным сахарным диабетом / Под ред. И.И. Дедова, М.В. Шестаковой, А.Ю. Майорова. 9-й вып. // Сахарный диабет. - 2019. - Т. 22. №S1-1. - C. 1-144. [Dedov II, Shestakova MV, Mayorov AYu, et al. Standards of specialized diabetes care. Ed. by Dedov II, Shestakova MV, Mayorov AYu. 9th edition. Diabetes mellitus. 2019;22(S1-1):1-144. (In Russ.)] doi: https://doi.org/10.14341/DM221S1

2. Erratum: Kidney Disease: Improving Global Outcomes (KDIGO) CKD-MBD Update Work Group. KDIGO 2017 clinical practice guideline update for the diagnosis, evaluation, prevention, and treatment of chronic kidney disease-mineral and bone disorder (CKD-MBD). Kidney Int Suppl (2011). 2017;7(3):1-59. doi: https://doi.org/10.1016/j.kisu.2017.04.001

3. Damm JA, Asbjornsdottir B, Callesen NF, et al. Diabetic nephropathy and microalbuminuria in pregnant women with type 1 and type 2 diabetes: prevalence, antihypertensive strategy, and pregnancy outcome. Diabetes Care. 2013;36(11):3489-3494. doi: https://doi.org/10.2337/dc13-1031

4. McCance DR, Holmes VA, Maresh MJ, et al. Vitamins $\mathrm{C}$ and $\mathrm{E}$ for prevention of pre-eclampsia in women with type 1 diabetes (DAPIT): a randomised placebocontrolled trial. The Lancet. 2010;376(9737):259-266. doi: https://doi.org/10.1016/s0140-6736(10)60630-7

5. Ekbom P, Damm P, Feldt-Rasmussen B, et al. Pregnancy outcome in type 1 diabetic women with microalbuminuria. Diabetes Care. 2001;24(10):1739-1744. doi: https://doi.org/10.2337/diacare.24.10.1739

6. Боровик Н.В., Потин В.В., Рутенбург Е.Л. Диабетические микрососудистые осложнения (ретинопатия и нефропатия) и беременность // Журнал акушерства и женских болезней. 2013. - T. 62. — №2. - C. 75-82. [Borovik NB, Potin BB, Rutenburg EL. Diabetic microvascular complications (retinopathy and nephropathy) and pregnancy. Journal of obstetrics and women's diseases. 2013;62(2):75-82. (In Russ.)]

7. Klemetti MM, Laivuori H, Tikkanen M, et al. Obstetric and perinatal outcome in type 1 diabetes patients with diabetic nephropathy during 1988-2011. Diabetologia. 2015;58(4):678-686. doi: https://doi.org/10.1007/s00125-014-3488-1

8. The Diabetes Control and Complications Trial Research Group. The effect of intensive treatment of diabetes on the development and progression of long-term complications in insulindependent diabetes mellitus. N Engl J Med. 1993;329(14):977-986. doi: https://doi.org/10.1056/NEJM199309303291401

9. Biesenbach $G$, Grafinger P, Stoger $H$, et al. How pregnancy influences renal function in nephropathic type 1 diabetic women depends on their pre-conceptional creatinine clearance. J Nephrol. 1999;12(1):41-46. doi: https://doi.org/10.1007/bf00400946

10. Reece EA, Leguizamon G, Homko C. Pregnancy performance and outcomes associated with diabetic nephropathy. Am J Perinatol. 1998;15(7):413-421. doi: https://doi.org/10.1055/s-2007-993968

11. The Diabetes Control and Complications Trial Research Group. Effect of pregnancy on microvascular complications in the diabetes control and complications trial. Diabetes Care. 2000;23(8):1084-1091. doi: https://doi.org/10.2337/diacare.23.8.1084

12. Verier-Mine $\mathrm{O}$, Chaturvedi $\mathrm{N}$, Webb $\mathrm{D}$, et al. Is pregnancy a risk factor for microvascular complications? The EURODIAB Prospective Complications Study. Diabet Med. 2005;22(11):1503-1509. doi: https://doi.org/10.1111/j.1464-5491.2005.01682.x

13. Reece EA, Coustan DR, Hayslett JP, et al. Diabetic nephropathy: pregnancy performance and fetomaternal outcome. Am J Obstet Gynecol. 1988;159(1):56-66. doi 10.1016/0002-9378(88)90494-2

14. Biesenbach G, Grafinger P, Zazgornik J, et al. Perinatal complications and three-year follow up of infants of diabetic mothers with diabetic nephropathy stage IV. Ren Fail. 2000;22(5):573-580. doi: https://doi.org/10.1081/jdi-100100898

15. Gordon M, Landon MB, Samuels $P$, et al. Perinatal outcome and long-term follow-up associated with modern management of diabetic nephropathy. Obstet Gynecol. 1996;87(3):401-409. doi: https://doi.org/10.1016/0029-7844(95)00420-3.

16. Imbasciati E, Gregorini G, Cabiddu G, et al. Pregnancy in CKD stages 3 to 5: fetal and maternal outcomes. Am J Kidney Dis. 2007;49(6):753-762. doi: https://doi.org/10.1053/j.ajkd.2007.03.022
17. Biesenbach G, Stöger H, Zazgornik J. Influence of pregnancy on progression of diabetic nephropathy and subsequent requirement of renal replacement therapy in female type I diabetic patients with impaired renal function. Nephrol Dial Transplant. 1992;7(2):105-109. doi: https://doi.org/10.1093/oxfordjournals.ndt.a092077

18. Purdy LP, Hantsch CE, Molitch ME, et al Effect of pregnancy on renal function in patients with moderate-to-severe diabetic renal insufficiency. Diabetes Care. 1996;19(10):1067-1074. doi: https://doi.org/10.2337/diacare.19.10.1067

19. Mackie AD, Doddridge MC, Gamsu HR, et al. Outcome of pregnancy in patients with insulin-dependent diabetes mellitus and nephropathy with moderate renal impairment. Diabet Med. 1996;13(1):90-96. doi: https://doi.org/10.1002/(SICI)10969136(199601)13:1<90::AID-DIA992>3.0.CO;2-M

20. Rossing $K$, Jacobsen $P$, Hommel $E$, et al. Pregnancy and progression of diabetic nephropathy. Diabetologia. 2002;45(1):36-41. doi: https://doi.org/10.1007/s125-002-8242-4

21. Piccoli GB, Clari R, Ghiotto S, et al. Type 1 diabetes, diabetic nephropathy, and pregnancy: a systematic review and meta-study. Rev Diabet Stud. 2013;10(1):6-26. doi: https://doi.org/10.1900/RDS.2013.10.6

22. Balsells M, Garcia-Patterson A, Gich I, et al. Maternal and fetal outcome in women with type 2 versus type 1 diabetes mellitus: a systematic review and metaanalysis. J Clin Endocrinol Metab. 2009;94(11):4284-4291. doi: https://doi.org/10.1210/jc.2009-1231

23. Bell R, Glinianaia SV, Tennant PW, et al. Peri-conception hyperglycaemia and nephropathy are associated with risk of congenital anomaly in women with pre-existing diabetes: a population-based cohort study. Diabetologia. 2012;55(4):936-947. doi: https://doi.org/10.1007/s00125-012-2455-y

24. Kerssen A, de Valk HW, Visser GH. Forty-eight-hour first-trimester glucose profiles in women with type 1 diabetes mellitus: a report of three cases of congenital malformation. Prenatal Diagnosis. 2006;26(2):123-127. doi: https://doi.org/10.1002/pd.1340

25. Cooper WO, Hernandez-Diaz S, Arbogast PG, et al. Major congenital malformations after first-trimester exposure to ACE inhibitors. N Engl J Med. 2006;354(23):2443-2451. doi: https://doi.org/10.1056/NEJMoa055202.

26. Doria A. Genetics of diabetes complications. Curr Diab Rep. 2010;10(6):467-475. doi: https://doi.org/10.1007/s11892-010-0147-x

27. Forbes JM, Coughlan MT, Cooper ME. Oxidative stress as a major culprit in kidney disease in diabetes. Diabetes. 2008;57(6):1446-1454. doi: https://doi.org/10.2337/db08-0057

28. Bramham K. Diabetic nephropathy and pregnancy. Semin Nephrol. 2017;37(4):362-369. doi: https://doi.org/10.1016/j.semnephrol.2017.05.008

29. Jensen DM, Damm P, Ovesen P, et al. Microalbuminuria, preeclampsia, and preterm delivery in pregnant women with type 1 diabetes: results from a nationwide Danish study. Diabetes Care. 2010;33(1):90-94. doi: https://doi.org/10.2337/dc09-1219

30. Bell R, Bailey K, Cresswell T, et al. Trends in prevalence and outcomes of pregnancy in women with pre-existing type I and type II diabetes. BJOG. 2008;115(4):445-452. doi: https://doi.org/10.1111/j.1471-0528.2007.01644.x

31. Hiilesmaa V, Suhonen L, Teramo K. Glycaemic control is associated with preeclampsia but not with pregnancy-induced hypertension in women with type I diabetes mellitus. Diabetologia. 2000:43(12):1534-1539. doi: https://doi.org/10.1007/s001250051565

32. Clausen P, Ekbom P, Damm P, et al. Signs of maternal vascular dysfunction precede preeclampsia in women with type 1 diabetes. J Diabetes Complications. 2007;21(5):288-293. doi: https://doi.org/10.1016/j.jdiacomp.2006.03.004

33. Мозговая Е.В. Диагностика и медикаментозная коррекция эндотелиальной дисфункции при гестозе: Автореф. дис. . докт. мед. наук. - СПб., 2004. — 40 с. [Mozgovaja EV. Diagnostika i medikamentoznaya korrektsiya endotelial'noi disfunktsii pri gestoze. [dissertation abstract] Saint Petersburg; 2004. 40 p. (In Russ.)]. Доступно по: https://search.rsl.ru/ru/record/01003244942. Ссылка активна на 14.04.2020.

34. Kelly CB, Hookham MB, Yu JY, et al. Subclinical first trimester renal abnormalities are associated with preeclampsia in normoalbuminuric women with type 1 diabetes. Diabetes Care. 2017;41(1):120-127. doi: https://doi.org/10.2337/dc17-1635 
35. Sandvik MK, Iversen BM, Irgens LM, et al. Are adverse pregnancy outcomes risk factors for development of end-stage renal disease in women with diabetes? Nephrol Dial Transplant 2010;25(11):3600-3607. doi: https://doi.org/10.1093/ndt/gfa275

36. American Diabetes Association. 14. Management of diabetes in pregnancy: standards of medical care in diabetes-2019. Diabetes Care. 2019;42(Suppl 1):S165-S172. doi: https://doi.org/10.2337/dc19-S014

37. Печерина Л.В. Эффективность низкомолекулярных гепаринов в комплексной терапии гестоза: Автореф. дис. ... канд. мед. наук. СПб., 2004. - 24 c. [Pecherina LV. Effektivnost' nizkomolekulyarnykh geparinov $\vee$ kompleksnoi terapii gestoza. [dissertation abstract] Saint Petersburg; 2004. 24 р. (In Russ.)]. Доступно по: https://search.rsl.ru/ru/record/01002726904. Ссылка активна на 14.04.2020.

38. Nielsen LR, DammP, MathiesenER. Improved pregnancy outcome in type1 diabetic women with microalbuminuria or diabetic nephropathy: effect of intensified antihypertensive therapy? Diabetes Care. 2009;32(1):38-44. doi: https://doi.org/10.2337/dc08-1526

39. Schaefer-Graf U, Napoli A, Nolan CJ. Diabetes in pregnancy: a new decade of challenges ahead. Diabetologia. 2018;61(5):1012-1021. doi: https://doi.org/10.1007/s00125-018-4545-y

40. Wahabi HA, Alzeidan RA, Esmaeil SA. Pre-pregnancy care for women with pre-gestational diabetes mellitus: a systematic review and meta-analysis. BMC Public Health. 2012;12:792. doi: https://doi.org/10.1186/1471-2458-12-792

41. Piccoli G, Zakharova E, Attini R, et al. Pregnancy in chronic kidney disease: need for higher awareness. A pragmatic review focused on what could be improved in the different CKD stages and phases. J Clin Med. 2018;7(1 1):415. doi: https://doi.org/10.3390/jcm7110415

42. Боровик Н.В., Ярмолинская М.И., Главнова О.Б., и др. Новые возможности использования цистатина С как предиктора ранней диагностики диабетической нефропатии // Журнал акушерства и женских болезней. - 2019. - Т. 68. - №3. C. 15-24. [Borovik NB, Yarmolinskaya MI, Gkavnova OB et al. Prospects of using cystatin $\mathrm{C}$ as an early predictor of diabetic nephropathy. Journal of obstetrics and women's diseases. 2019;68(3):15-24. (In Russ.)] doi: https://doi.org/10.17816/JOWD68315-24

43. Li DK, Yang C, Andrade S, et al. Maternal exposure to angiotensin converting enzyme inhibitors in the first trimester and risk of malformations in offspring: a retrospective cohort study. BMJ. 2011;343:d5931. doi: https://doi.org/10.1136/bmj.d5931

44. Bullo M, Tschumi S, Bucher BS, et al. Pregnancy outcome following exposure to angiotensin-converting enzyme inhibitors or angiotensin receptor antagonists: a systematic review. Hypertension. 2012;60(2):444-450. doi: https://doi.org/10.1161/HYPERTENSIONAHA.112.196352

45. Diabetes in pregnancy: management of diabetes and its complications from preconception to the postnatal period. NICE guideline 3 [cited 26 August 2015]. National Institute of Clinical and Health Excellence; 2015. Available from: https://www.nice.org.uk/guidance/ng3.

46. Carr DB, Koontz GL, Gardella C, et al. Diabetic nephropathy in pregnancy: suboptimal hypertensive control associated with preterm delivery. Am J Hypertens. 2006;19(5):513-519. doi: https://doi.org/10.1016/j.amjhyper.2005.12.010

47. Nielsen LR, Muller C, Damm P, et al. Reduced prevalence of early preterm delivery in women with type 1 diabetes and microalbuminuria: possible effect of early antihypertensive treatment during pregnancy. Diabet Med. 2006;23(4):426-431. doi: https://doi.org/10.1111/j.1464-5491.2006.01831.x

48. Fullerton B, Jeitler K, Seitz M, et al. Intensive glucose control versus conventional glucose control for type 1 diabetes mellitus. Cochrane Database Syst Rev. 2014;2014(2):CD009122. doi: https://doi.org/10.1002/14651858

49. Kähler P, Grevstad B, Almdal T, et al. Targeting intensive versus conventional glycaemic control for type 1 diabetes mellitus: a systematic review with meta-analyses and trial sequential analyses of randomised clinical trials. BMJ Open. 2014;4(8):e004806. doi: https://doi.org/10.1136/bmjopen-2014-004806
50. Shurraw S, Hemmelgarn $B$, Lin $M$, et al. Association between glycemic control and adverse outcomes in people with diabetes mellitus and chronic kidney disease: a populationbased cohort study. Arch Intern Med. 2011;171(21):1920-1927. doi: https://doi.org/10.1001/archinternmed.2011.537

51. Diabetes Control and Complications Trial Research Group. The effect of intensive treatment of diabetes on the development and progression of long-term complications in insulindependent diabetes mellitus. N Engl J Med. 1993;329(14):977-986. doi: https://doi.org/10.1056/NEJM199309303291401

52. Газизова Г.Р. Маркеры воспаления диабетической нефропатии у беременных с сахарным диабетом 1 типа: Автореф. дис. ... канд. мед. наук. - СПб., 2015. - 22 с. [Gazizova GR. Markery vospaleniya diabeticheskoy nephropatii u beremennykh s sacharnym diabetom 1 tipa. [dissertation abstract] Saint Petersburg; 2015. 22 p. (In Russ.)]. Доступно по: https://search.rsl.ru/ru/record/01005570273. Ссылка активна на 14.04.2020.

53. Brown JS, Wessells $\mathrm{H}$, Chancellor MB, et al. Urologic complications of diabetes. Diabetes Care. 2005;28(1):177-185. doi: https://doi.org/10.2337/diacare.28.1.177

54. Wessells $\mathrm{H}$, Braffett $\mathrm{BH}$, Holt SK, et al. Burden of urological complications in men and women with long-standing type 1 diabetes in the diabetes control and complications trial/epidemiology of diabetes interventions and complications cohort. Diabetes Care. 2018;41(10):2170-2177. doi: https://doi.org/10.2337/dc18-0255

55. Mazor-Dray E, Levy A, Schlaeffer F, Sheiner E. Maternal urinary tract infection: is it independently associated with adverse pregnancy outcome? J Matern Fetal Neonatal Med. 2009:22(2):124-128. doi: https://doi.org/10.1080/14767050802488246

56. Meuwese $\mathrm{CL}$, Carrero JJ. Chronic kidney disease and hypothalamic-pituitary axis dysfunction: the chicken or the egg? Arch Med Res. 2013;44(8):591-600 doi: https://doi.org/10.1016/j.arcmed.2013.10.009

57. Finkelstein FO, Shirani S, Wuerth D, et al. Therapy insight: sexual dysfunction in patients with chronic kidney disease. Nat Clin Pract Nephrol. 2007;3(4):200-207. doi: https://doi.org/10.1038/ncpneph0438

58. Приказ Министерства здравоохранения и социального развития Российской Федерации № 736 от 3 декабря 2007 г. «Об утверждении перечня медицинских показаний для искусственного прерывания беременности». [Order № 736 of the Ministry of health and social development of Russian Federation «Ob utverzhdenii perechn'a meditsinskikh pokazaniy dl'a iskusstvennogo preryvaniya beremennosti», dated 2007 December 3. (In Russ.)]. Доступно по: https://www.rosminzdrav. ru/documents/7780-prikaz-minzdravsotsrazvitiya-rossii-736-ot-3dekabrya-2007-g. Ссылка активна на 14.04.2020.

59. Piccoli GB, Minelli F, Versino E, et al. Pregnancy in dialysis patients in the new millennium: a systematic review and metaregression analysis correlating dialysis schedules and pregnancy outcomes. Nephrol Dial Transplant. 2016;31(11):1915-1934. doi: https://doi.org/10.1093/ndt/gfv395

60. Ibarra-Hernandez M, Alcantar-Vallin M, Soto-Cruz A, et al. Challenges in managing pregnancy in underserved women with chronic kidney disease. Am J Nephrol. 2019;49(5):386-396. doi: https://doi.org/10.1159/000499964

61. Webster P, Lightstone L, McKay D, et al. Pregnancy in chronic kidney disease and kidney transplantation. Kidney Int. 2017;91(5):1047-1056. doi: https://doi.org/10.1016/j.kint.2016.10.045

62. Cabiddu G, Spotti D, Gernone G, et al. A best-practice position statement on pregnancy after kidney transplantation: focusing on the unsolved questions. The Kidney and Pregnancy Study Group of the Italian Society of Nephrology. J Nephrol. 2018;31(5):665-681. doi: https://doi.org/10.1007/s40620-018-0499-x

63. Kidney Disease: Improving Global Outcomes (KDIGO) Transplant Work Group. KDIGO clinical practice guideline for the care of kidney transplant recipients. Am J Transplant. 2009;9(Suppl 3):1-155. doi: https://doi.org/10.1111/j.1600-6143.2009.02834.x 


\section{ИНФОРМАЦИЯ ОБ АВТОРАХ [AUTHORS INFO]}

*Шилова Екатерина Сергеевна [Ekaterina S. Shilova]; адрес: Россия, 199034 Санкт-Петербург, Менделеевская линия, д. 3 [address: 3, Mendeleyevskaya Line, 199034 St. Petersburg, Russian Federation]; ORCID: https://orcid.org/0000-0002-5225-6054; eLibrary SPIN: 9703-5970; e-mail: katia.shilova@gmail.com

Боровик Наталья Викторовна, К.M.H. [Natalya V. Borovik, MD, PhD]; ORCID: https://orcid.org/0000-0003-0835-6741; eLibrary SPIN: 9010-7276; e-mail: borovik1970@yandex.ru

Ярмолинская Мария Игоревна, д.м.н., профессор [Maria I. Yarmolinskaya, MD, PhD, Professor];

ORCID: https://orcid.org/0000-0002-6551-4147; eLibrary SPIN: 3686-3605; e-mail: m.yarmolinskaya@gmail.com

\section{ЦИТИРОВАТЬ:}

Шилова Е.С., Боровик Н.В., Ярмолинская М.И. Диабетическая нефропатия у беременных с сахарным диабетом 1 типа, современный взгляд на проблему // Сахарный диабет. — 2020. — Т. 23. — №4. — С. 340-348. doi: https://doi.org/10.14341/DM12228

\section{TO CITE THIS ARTICLE:}

Shilova ES, Borovik NV, Yarmolinskaya MI. Diabetic nephropathy in type 1 diabetes and pregnancy. Modern view of the problem. Diabetes Mellitus. 2020;23(4):340-348. doi: https://doi.org/10.14341/DM12228 